\title{
Development of an Iot Based Energy Management System for Air Handling Unit
}

\author{
Ananda Krishnan B, Aswin Kumar P, Shilpa VK, Joselin Retna Kumar G
}

\begin{abstract}
The emergence of automation and smart power management systems pave to create a better energy management system. Measuring energy savings and potential reduction of peak demand in a building due to adjustment of AHU (Air Handling Unit) setpoint during working hours is critical for ideal demand-side allocations. Since Air Handling Units are one of the major energy consuming system in a building. This has been a great challenge given too many variable physical characteristics and $\mathrm{AHU}$ system thermal dynamics in commercial buildings. Based on the building model, developed and authenticate against measured power consumption data from a smart energy meter, this project deals with how energy savings and peak demand reduction potentials of a building are impacted by $A H U$, setpoint changes during a working period. It is of the utmost necessity to make a prototype for an existing $\mathrm{AHU}$ in order to minimize wastage of power in older buildings without any major renovation. This can be achieved by making the existing system into a closed loop system by our model prototype.
\end{abstract}

Keywords: AHU, peak demand, power consumption, closed loop system

\section{INTRODUCTION}

In order to better manage the energy demand and increase building energy efficiency, and intelligent building should offer a smart approach in order to reduce power usage and work efficiently. A clever structure frequentl utilizes a structure computerization framework (Building Management System) to screen and ideally control different structure loads, for example, lighting and warming, ventilating, and air molding frameworks. Building Management System is integrated control and monitor of hardware and software, under a platform connecting heating, venting and air conditioning system (HVAC), security, lighting, and many other systems. BAS is purpose is to increase the human comfort inside the building to increase in protectivity and wellbeing of the employee.

Revised Manuscript Received on April 09, 2020.

* Correspondence Author

Ananda Krishnan B*, Department of Electronics and Instrumentation Engineering, SRM Institute of Science and Technology, Chengalpattu, Tamil Nadu, India. Email: akbn1998@gmail.com

Aswin Kumar P, Department of Electronics and Instrumentation Engineering, SRM Institute of Science and Technology, Chengalpattu, Tamil Nadu, India. Email: askeie043@gmail.com

Shilpa VK, Department of Electronics and Instrumentation Engineering, SRM Institute of Science and Technology, Chengalpattu, Tamil Nadu, India. Email: shilpavalsa23@gmail.com

Dr. Joselin Retna Kumar G, Department of Electronics and Instrumentation Engineering, SRM Institute of Science and Technology, Chengalpattu, Tamil Nadu, India. Email: joselinrk@gmail.com

(C) The Authors. Published by Blue Eyes Intelligence Engineering and Sciences Publication (BEIESP). This is an open access article under the CC BY-NC-ND license (http://creativecommons.org/licenses/by-nc-nd/4.0/) consist of Field level controllers and Server level controller to monitor and control the systems inside and outside the building. Building Management System allows a centralized control for all systems to minimize the human intervention. An Air Handling Unit (AHU) uses heating coil and cooling coil to heat or cooldown the air that is passed through them depending on the environment. A humidifier is also the part of the AHU which maintains the humidity of the air within human comfort parameter. A centrifugal pump id used to create pressure difference to circulate the conditioned air. A motor is used to drive the pump. Filters are used to filter the air before it is conditioned.Air Handling Unit (AHU) is one of the major power consuming load in a building by reducing the known required task of the AHU system the energy can be saved. This could be achieved by reducing the working hours' usage and by shutting down the Fan Coil Unit (FCU) when the setpoint has been reached. This prototype also consists of a power and energy measurement system which helps to save energy by measuring and storing how much energy consumed per day. We could analyze the data from energy meter to optimize the energy need of the load or we could upgrade/replace with energy efficient load.

\section{LITERARY SURVEY}

There are different methods and other possible ways that has been mentioned, which are:

The initials are based on different concept one of them is green buildings on AC. Thermal balance based. Algerian is used to find the efficiency on AC. It gives a comparison between the construction and thermal balance with and without the usage of the plants[4]. Artificial climate for cooling/heating by checking the various parameters of thermal comfort temperature, humidity, purity of air and air speed. It uses clean technologies not polluting as the magnetic refrigeration, the thermoelectric refrigeration.

With plants it gives a better impact, not only through reduction of the power but also it gives the positive impact on the people who are working on them.

Other study shows the impact of controllers in energy management. The control parameters are PID, that is being used in order to ON and OFF the fan coil unit (FCU) during the working hours only [3]. It is done through the linear system design which requires mathematical algorithm in order to control the system. Without reducing the size of the equipment and the design, a secondary side voltage of the temperature sensor. Another method is about multi input and multi output (MIMO), that is MIMO approach for analyses and control system designs [2]. IDA indoor climate and energy ICE simulation programs are being used. The inputs are air flow temperature and hot water temperature, 
the disturbance is environmental input of outdoor temperature, wind direction and velocity. Outputs are temperature and humidity. It gives an approx. good results when the hot water and electrical component are being compared to the measured values. The outdoor temperature generally gives a huge impact. It has 2 state space model of MIMO, 3 variables, 2 inputs and 3 outputs with an acceptable range. Apart from these, how much energy savings and peak demand reduction potential of a building are impacted by AHU set point changes during the desired period is also considered. The simulation results show that during high outdoor temperature are expected to achieve a predictable percentage of daily energy savings and peak load reduction when AHU set is taken into consideration. EQUEST, building mode and validated against hourly electricity consumption data from smart meter [1]. In other words, with the previous data values, we can predict the values. Power consumption of AHU is the same but the only difference is that when it subjects to outdoor temperature. To solve this problem, we use drive the model in relation with outdoor humidity. By measuring it gives an idea that the outdoor temperature is directly proportional to the energy consumption. That is when the outdoor temperature increases the energy consumption increases [5][6].

\section{PROPOSED ARCHITECTURE}

The fig.3.1 shows the block diagram representation of our proposed working model includes the programming module, control module, the on/off controlling relays, power \& energy sensor and temperature sensors. As the core of the control circuit, the raspberry $\mathrm{Pi}$ is responsible for data acquisition, signal output, algorithm operation and so forth.

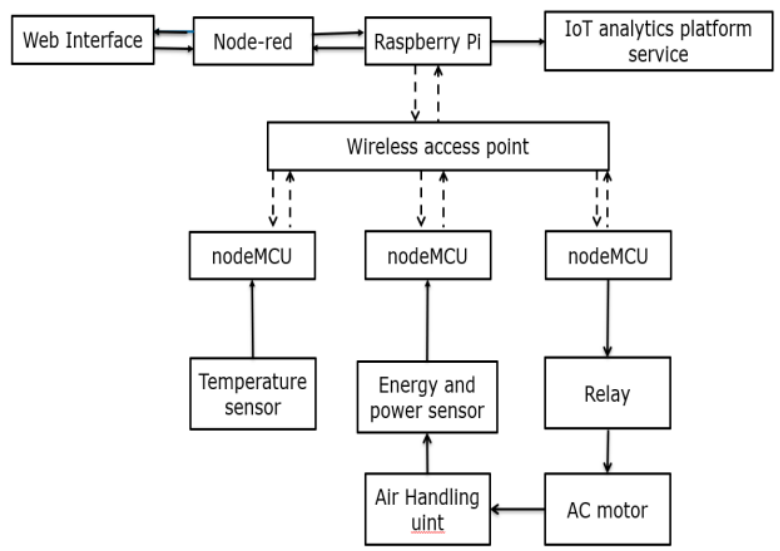

Fig.3.1 System model structure

There are three kinds of connections between the raspberry $\mathrm{Pi}$ through wireless access point, where one kind is the acquisition of temperature data from a different locations, one kind is to receive data from power and energy sensor, and the rest one is output control signal, the function of which is to on or off the fan coil unit.

MQTT protocol is very fast, allowing communication between RaspberryPi and nodeMCU without any delay. Hyper Text Transfer Protocol (HTTP) being used on request-response isn't the option for transferring data fast and proficiently.

\section{A. MOSQUITTO BROKER:}

Mosquitto is an open source software that is developed for non-TCP communication between IoT gateways and cloud platform. Mosquitto acts as communication platform between NodeMCU and Raspberry pi.

The main benefits of the mosquito broker are that it eliminates insecure connections, easily scales, manages connect client states and reduces network strain.

\section{B. SENSORS AND RELAY CONNECTIONS}

In this project we integrate the Relay and Multiple temperature sensor for the nodeMCU and ESP01 board with Node-RED. The ESP8266 and nodeMCU will establish an MQTT connection with a Raspberry Pi running Node-RED and Mosquitto MQTT broker.

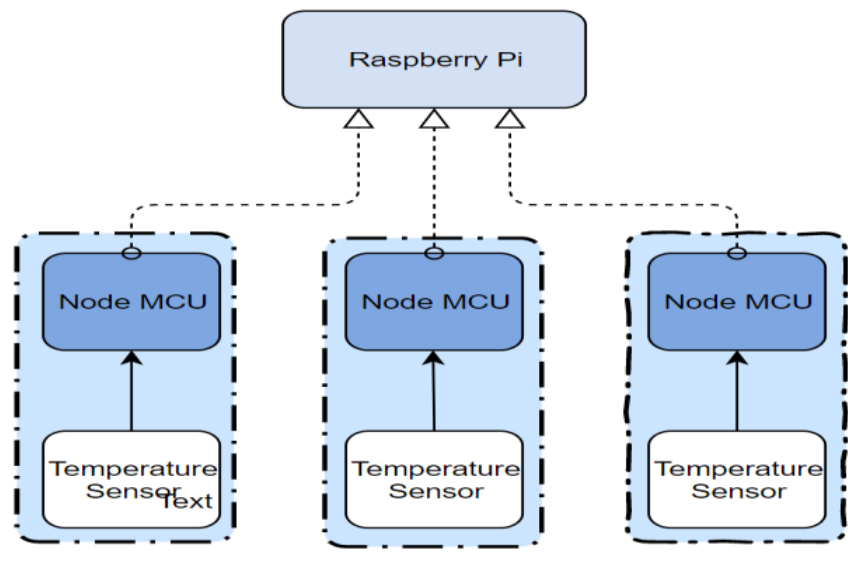

Fig.3.2.1 Temperature sensing node system structure

In the above Fig3.2.1, multiple nodeMCU and esp01 boards is connected to the pi through Wi-Fi. Each nodeMCU and ESP01 is connected with a ds13b20 temperature sensor. DS18B20 is digital temperature sensor that uses single wire interface, which requires only one data line for communication with NodeMCU.

\section{IMPLEMENTATION}

The comprehensive implementation details can be visualized as shown in Fig.4.1

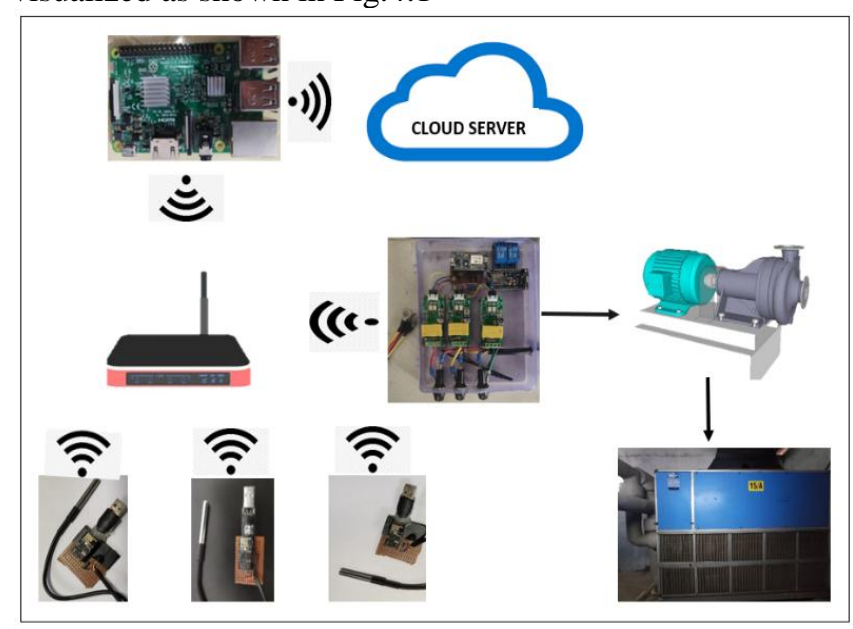

Fig.4.1 prototype implementation

The details about the Algorithm implementation are described below. 
Step1: The first step is to deal with Raspberry Pi to make it as wireless access point.

Step2: Then the second step is to connect nodes to raspberry pi for the acquisition of temperature data from sensors which are placed across the floor in building to transmit and receive the temperature data.

Step3: Programming the set point in the system.

Step4: The temperature sensor nodes sense the room temperature, send the data to controller and then controller compare it with set point.

Step5: When the set point is achieved the controller cut down the compressor power which in turn stop the circulation of conditioned air in the room. When the temperature inside the conditioned room is increased the temperature, sensor senses it and the controller turn on the compressor, flow diagram of controlling part is given in fig4.1.1.

Step6: The energy readings of the motor are sent to the central controller for further analysis and data management.

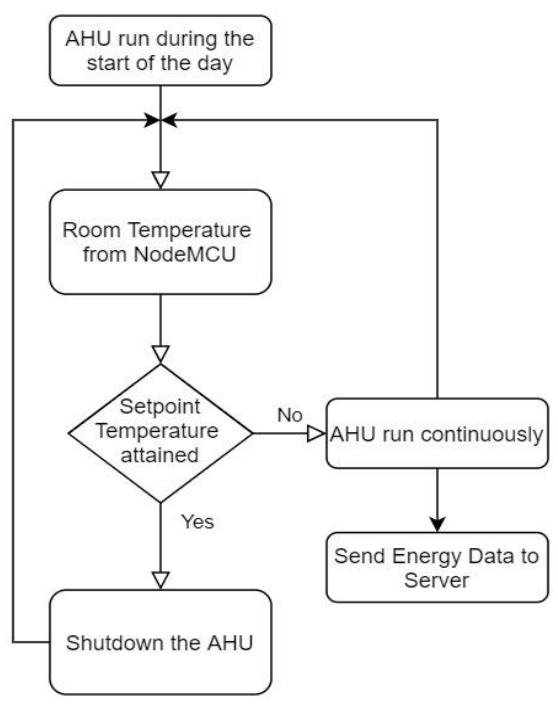

Fig 4.1.1 Flow diagram of control

In some cases, the temperature where the compressor is turned on and off is so close that the motor will begin to cycle on and off rapidly. This action is called short cycling and it can be harmful to the compressor. The difference between the point where the compressor is turned on and where the compressor is turned off is called the differential gap and the system algorithm can be modified to allow the differential gap to be fully adjustable. By introducing differential gap into the system helps to increase the life term of the Motor.

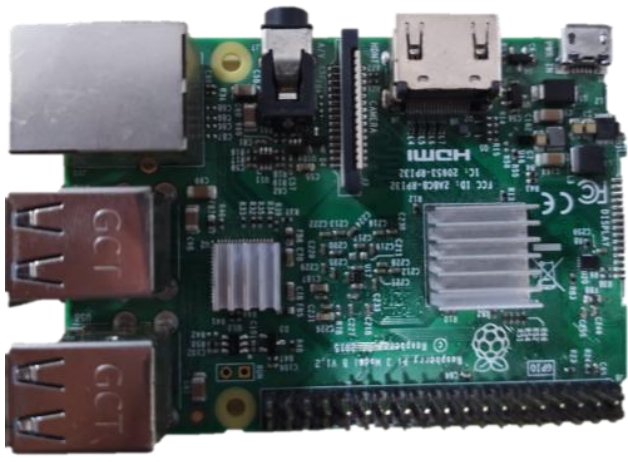

Fig.4.2 Raspberry pi
Setting up your local and secure 'Internet of Things' (IoT) server is easy with Mosquitto, NodeRed and a Raspberry Pi Fig.4.2. MQTT broker such as mosquitto on the same Raspberry Pi that Node-RED is running on. NodeRED is a programming tool and create live dashboards.

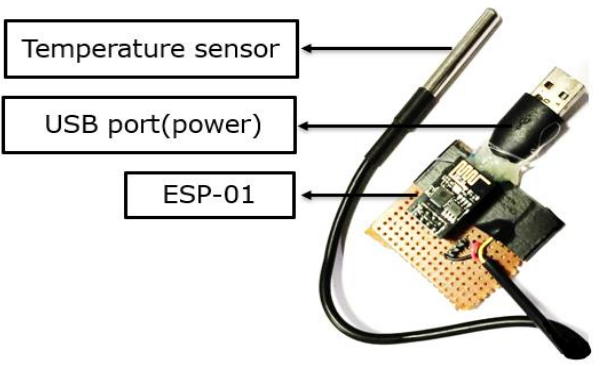

Fig.4.3 Temperature sensor node

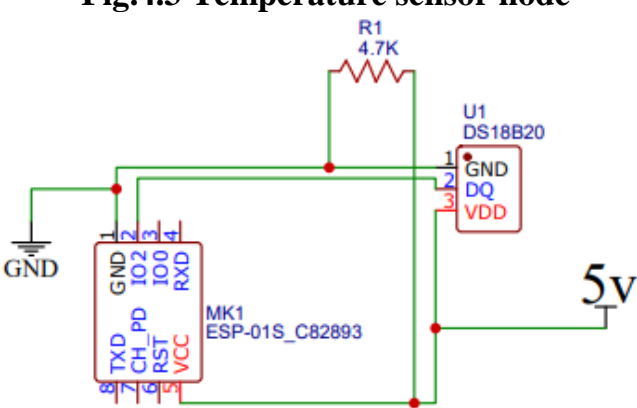

Fig.4.3.1 Circuit schematic of temperature sensor node

Temperature sensor is connected to general purpose input and output port of the ESP-01. Temperature data is delivered to Raspberry pi through MQTT broker. Based on the number of rooms, the number of temperature sensor node can be placed. During testing we used three individual temperature sensor nodes can be placed. Above fig. 4.3 is a temperature sensor node whose internal schematic are shown in Fig.4.3.1.

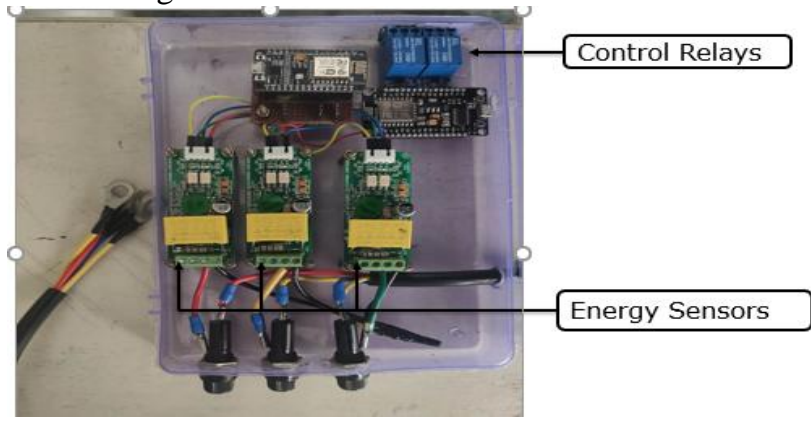

Fig.4.4 3-Phase Energy monitoring and control relay module

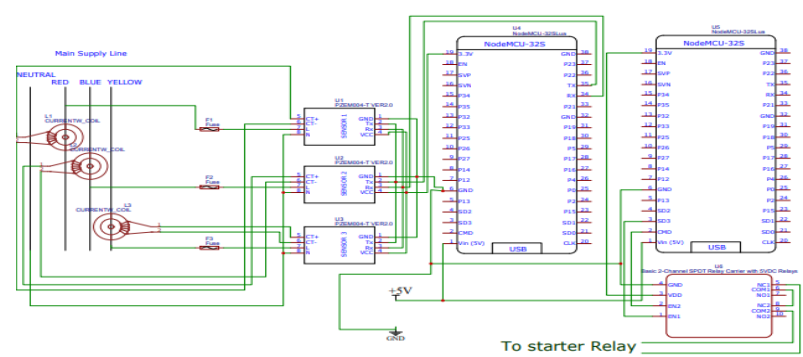

Fig.4.4.1 Circuit schematics of Energy monitoring and control relays

Published By:

Blue Eyes Intelligence Engineering

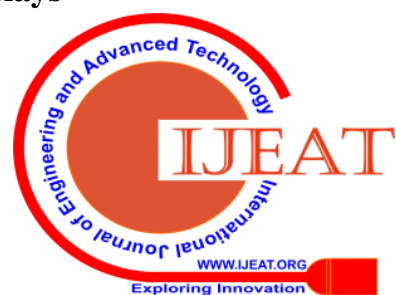


Energy and power sensing module that is interfaced using NodeMCU. This measures voltage, current, frequency and energy and delivers data to a MQTT broker.

We can measure true 3-phase energy monitoring system, along with relays that control Air handling Unit which is shown in Fig.4.4 whose internal schematics are shown in Fig.4.4.1. The energy data from the energy meter is delivered through Wi-Fi access point to raspberry Pi.

\section{RESULTS AND DISCUSSION}

Using Nodered, we created graphical user interface as show in below fig 5.1 from which setpoint can be given to the system and used to display temperature of temperature sensor node placed in different rooms.

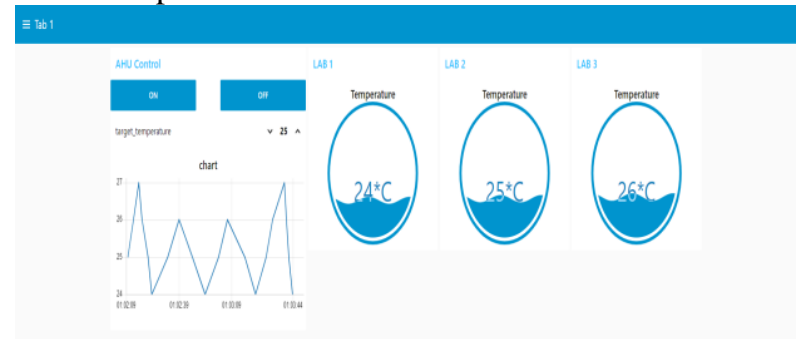

Fig.5.1 Graphical user interface for displaying room temperature

Below fig 5.2 shows displays voltage, current, frequency, power and energy of 3 phase supply. All the values displaying through graphical user interface is real time value and all those values are also stored in cloud storage platform.

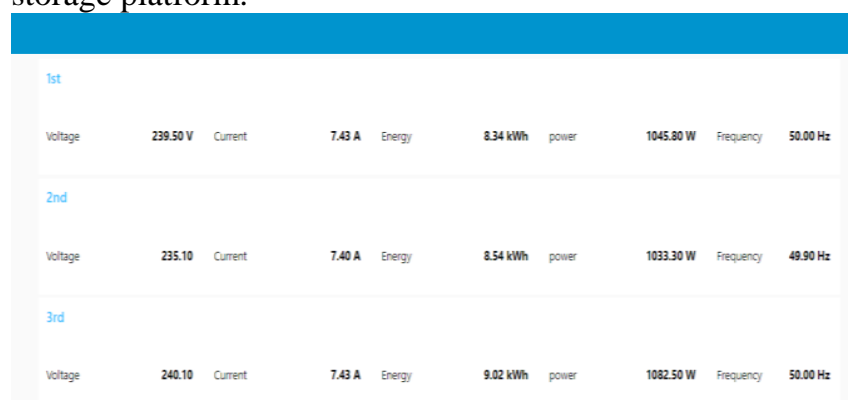

Fig.5.2 Graphical user interface for displaying 3-Phase energy monitoring system

\section{A. Without the Implementation of our Solution}

The given energy consumed by the AHU without implementing the model. The mentioned data are the measurements of the energy taken for a day during working hours (9AM-5PM). The total energy consumption is 27.24 kWh for a day for AHU unit.

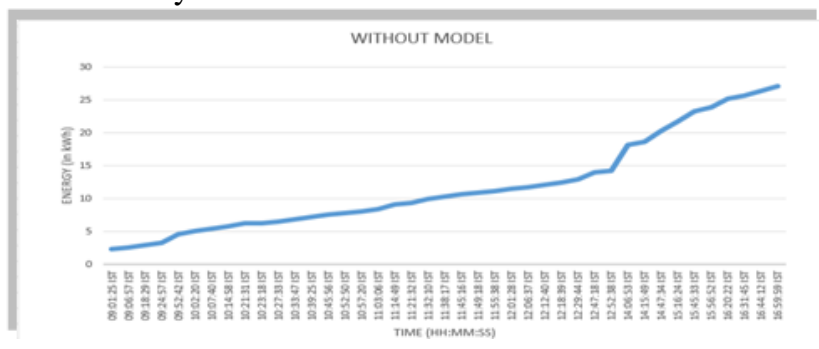

Fig.5.1.1 Energy consumption without implementing the model

Published By:

Blue Eyes Intelligence Engineering \& Sciences Publication power cut during the test run. for a day for one AHU unit. the Air handing unit to maintain the temperature is linear, sudden rise in the graph is due to high current drawn after

\section{B. With the Implementation of our Solution}

In this case, the relays are connected parallel to the DOL starter Relays so that the AHU can be turned on \& off automatically based on setpoint given to the system. The given energy consumed by the AHU with implementation of the proposed model. The mentioned data are the measurement of the energy taken for a day during working hours (9AM-5PM). The total energy consumption is $21 \mathrm{kWh}$

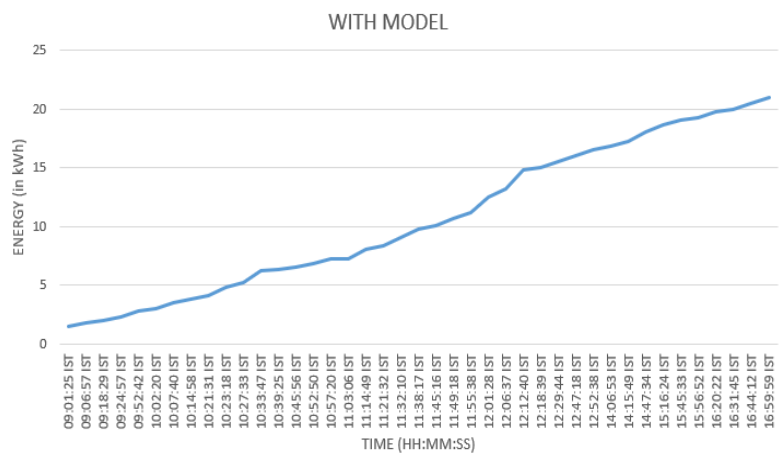

Fig.5.2.1 Energy consumption without implementing the model

From the above graph fig.5.2.1 we can see the energy utilized by the Air handling unit after implement close loop system. And also, with the close loop system installed the energy consumption is further reduced it consumes about 6$8 \mathrm{kWh}$ less than the pre-existing method.

\section{CONCLUSION}

The below fig 6.1 graph shows the comparison between open and closed loop, as you can see there's a difference in the power usage when it is a closed loop.

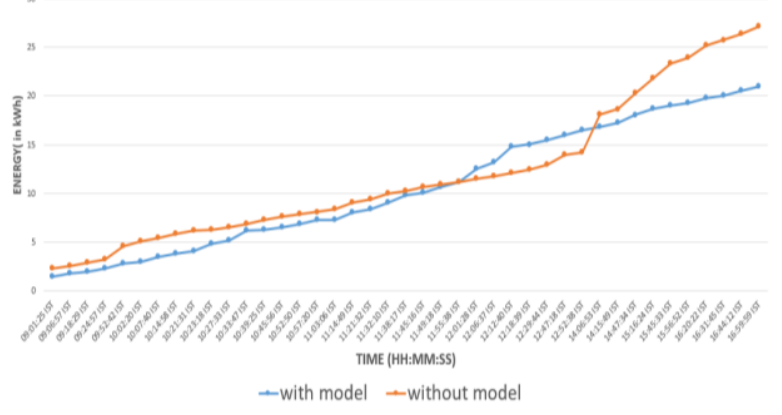

Fig 6.1 Comparing Energy consumption without and with implementing the model 
Table 6.1 Comparing total Energy consumption without and with implementing the model

\begin{tabular}{|l|c|c|}
\hline & $\begin{array}{c}\text { Without model(open } \\
\text { loop system) }\end{array}$ & $\begin{array}{c}\text { With model(closed loop } \\
\text { system) }\end{array}$ \\
\hline $\begin{array}{l}\text { Total Energy } \backslash \text { day } \\
(\mathrm{kWh})\end{array}$ & 27 & 21 \\
\hline
\end{tabular}

Thereby, from table 6.1 with our proposed closed loop system consume less energy than the pre-existing open loop system. This helps in reducing the power consumption therefore reduction in the cost of the electricity bill.

Therefore, in this paper we've developed a model for the operation of saving the energy when it is connected through closed loop system. In the developing countries, it is important to save the energy consumption not only to reduce the cost but also for the better and healthier environment for the present and future environment. The proposed system optimizes the with and without model accumulated over the time. Therefore, by the proposed module ie., closed loop system it saves energy.

\section{REFERENCES}

1. Quang Duy La, Member, IEEE, Yiu Wing Edwin Chan," Power Management of Intelligent Buildings Facilitated by Smart Grid: A Market Approach" IEEE TRANSACTIONS ON SMART GRID, VOL. 7, NO. 3, MAY 2016.

2. Tariq Iqbal, Almahdi Abdo-Allah, Kevin Pope, "Modeling and Analysis of an AHU System for the S.J. Carew Building at Memorial University" 2017 IEEE 30th Canadian Conference on Electrical and Computer Engineering (CCECE).

3. Long Meng, Xingxuan Wang," Intelligent Controllers Design Based on Electronic Temperature Controllers for Large Central AirConditioning Systems" Proceedings of the 36th Chinese Control Conference July 26-28, 2017, Dalian, China.

4. Younes Chiba, Yacine Marif, Abdelali Boukaoud, Laid Meredef, Youcef Kaidi, "Impact of green buildings on AHU system located in Algeria" 2018 International Conference on Applied Smart Systems (ICASS'2018) 24-25 November 2018, Médéa, ALGERIA.

5. Mengmeng Cai, Sneharaj Ramdaspalli, Manisa Pipattanasomporn, Saifur Rahman, Ahmadreza Malekpour and Sri Raghavan Kothandaraman," Impact of AHU Set Point Adjustment on Energy Savings and Peak Load Reductions in Buildings" IEEE 2018.

6. Xiangyu Zhang, Mengmeng Cai, Manisa Pipattanasomporn, Saifur Rahman," A Power Disaggregation Approach to Identify Power Temperature Models of AHU Units” IEEE 2018.

\section{AUTHORS PROFILE}

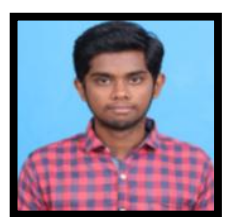

Ananda Krishnan B became a Member of IET in 2016. Anand was born in Chengalpattu, Tamilnadu, India. He is currently pursuing Bachelor's Degree B. Tech in the stream of Electronics and Instrumentation Engineering from SRM Institute of Science and Technology, Chengalpattu, India. He also participated in many inter college technical competition like Smart India Hackathon which is a national level hackathon, also participated in Makeathon VIT, Vellore and an honored member of Recon Subsea Team whose primarily goal to build and compete with underwater submarines. He also trained in basics of embedded system design from TVS training institute Chennai.

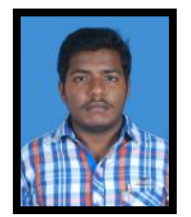

Aswin Kumar $\mathbf{P}$ became a Member of IET in 2016 Aswin was born in Thoothukudi, Tamilnadu, India. He is currently pursuing Bachelor's Degree B. Tech in the stream of Electronics and Instrumentation Engineering from SRM Institute of Science and Technology, Chengalpattu, India. He also participated in many inter college technical competition like Smart India Hackathon which is a national level hackathon, also participated in Makeathon VIT, Vellore. He also trained in basics of embedded system design from TVS training institute Chennai. Chennai.

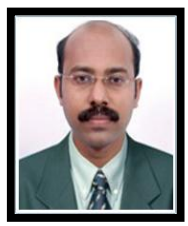
2016.She was born in Ooty, Tamil Nadu, India. She's currently pursuing her bachelor's degree in Electronics and Instrumentation Engineering from SRM Institute of Science and Technology, Chengalpattu, Tamil Nadu. She has participated in MUNs and other college fest and has volunteered for the NGO, blooming beacon. Apart from this, she has been an active member in the aero modelling club. She also trained in basics of embedded system design from TVS training institution

Dr. Joselin Retna Kumar G is working as Associate Professor at Department of Electronics and Instrumentation Engineering, SRM IST. He had completed his bachelor's in Electrical and Electronics Engineering from Manonmanium Sundaranar University, in 2002 and Master's in Process control and Instrumentation from Annamalai University, in 2004. He also got his doctorate in Electronics Engineering from Sathyabama University, in 2014. He also published various papers in topics of Automation, Signal processing etc. He is also a Quarter finalist in DST \& Texas Instruments India Innovation Challenge Design Contest 2017.

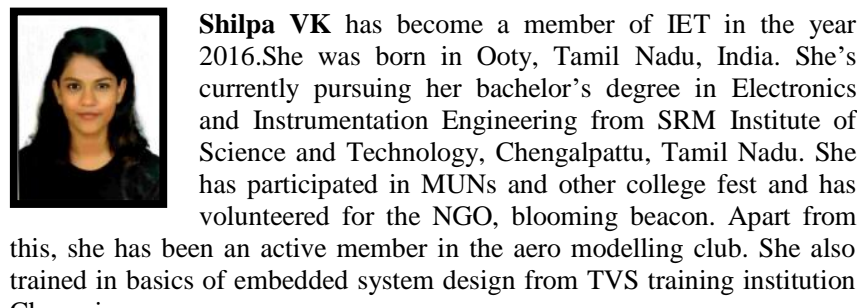

\section{.}

